a dose-dependent and time-dependent manner, with maximal effect at a concentration of $10^{-8} \mathrm{~mol} / \mathrm{l}$ at $12 \mathrm{~h}$ (in the level of protein secretion from the cells, $\mathrm{p}<0.01$ ) or $24 \mathrm{~h}$ (in the level of protein expression in the cells, $\mathrm{p}<0.01$ ), which could also be inhibited by these inhibitors ( $p<0.01$ in all groups).

Conclusion Urotensin II may stimulate the expression of monocyte chemoattractant protein-1 in rat aortic adventitial fibroblasts, through its receptor and the $\mathrm{Ca}^{2+}$ channel, protein kinase $\mathrm{C}$, mitogen-activated protein kinase, calcineurin and Rho kinase signal transduction pathways, contributing to the vascular inflammation.

\section{e0157 RNA INTERFERENCE TARGETING ACE AND AT1R GENE REDUCED BLOOD PRESSURE AND IMPROVED MYOCARDIAL REMODELLING IN SHR}

doi:10.1136/hrt.2010.208967.157

Zhou Hua, Gao Fen, Bian Yunfei, Li Maolian, Oiu Ling, Wu Weidong, He Junhua, Xiao Chuanshi. The Second Hospital of Shanxi Medical University, Taiyuan, Shanxi, China

Introduction Angiotensin-converting enzyme (ACE) and angiotensin II (Ang II) Type 1 receptor (ATIR) have been shown to play an important role in the pathogenesis of hypertension.

Objective To investigate the effects of RNA interference (RNAi) ATIR and ACE on blood pressure and myocardial hypertrophy in spontaneously hypertensive rats (SHR).

Methods SHRs were treated with normal saline as vehicle controls, with Ad5-EGFP as vector controls, with recombinant adenoviral vectors Ad5-EGFP-ACE-shRNA carrying shRNA for ACE as ACERNAi, Ad5-EGFP-AT1R-shRNA carrying shRNA for AT1R as AT1RRNAi, and Ad5-EGFP-ACE-AT1R-shRNA carrying shRNA for ACE and AT1R as ACE-AT1R-RNAi. WKY rats were taken as normotensive controls treated with normal saline. Systolic blood pressure of the caudal artery was recorded. Serum levels of ACE and Ang II were determined with ELISA. ACE and AT1R mRNA and protein level were determined in myocardium, aorta, kidney and lung. On day-40 of the experiment, heart was pathologically and ultrastructurly examined. The ratio of heart weight to and left ventricular weight to body weight were calculated.

Results Serum concentration of ACE was lower in ACE-RNAi rats, AT1R-RNAi rats and ACE-AT1R-RNAi rats respectively than in vehicle and vector controls (both $\mathrm{p}<0.05$ ). Serum concentration of Ang II was significantly lower in ACE-RNAi rats and higher in AT1R-RNAi rats than in vehicle and vector controls $(p<0.05)$. The expressions of ACE and AT1R mRNA and protein were significantly reduced in the myocardium, aorta, kidney and lung in ACE-RNAi rats, AT1R-RNAi rats and ACE-AT1R-RNAi rats respectively, compared with that in vehicle and vector controls (all $p<0.05$ ). ACE-RNAi, AT1R-RNAi and ACE-AT1R-RNAi treatments resulted in a reduction of systolic blood pressure by $(22 \pm 6) \mathrm{mm} \mathrm{Hg}$, $(20 \pm 5) \mathrm{mm} \mathrm{Hg},(23 \pm 7) \mathrm{mm} \mathrm{Hg}$ respectively and the reduction lasted for more than 15 days. In contrast, blood pressure was continuously increased in the vehicle controls as well as vector controls. The ratio of heart weight to and left ventricular weight to body weight were significantly lower in ACE-RNAi rats, AT1RRNAi rats and ACE-AT1R-RNAi rats respectively than in the vehicle $(p<0.05)$ and vector controls $(p<0.05)$. Myocardial pathology and ultrastructure were also significantly improved in ACE-RNAi rats, AT1R-RNAi rats and ACE-AT1R-RNAi rats, compared with that in vehicle and vector controls.

Conclusions ACE and AT1R silencing had significant antihypertensive effects and reversed hypertensive-induced cardiac hypertrophy in SHR, so RNAi might be a new strategy in control hypertension.

\section{e0158 TRANSIENT PREHYPERTENSIVE TREATMENT IN SPONTANEOUSLY HYPERTENSIVE RATS: A COMPARISON OF LOSARTAN AND AMLODIPINE REGARDING LONG-TERM BLOOD PRESSURE AND CARDIAC PROTECTION}

doi:10.1136/hrt.2010.208967.158

Jinxiu Lin, Liming Lin, Hong Tang, Changsheng Xu. Department of Cardiology, The First Affiliated Hospital of Fujian Medical University, Fujian Hypertension Research Institute, Fuzhou, China

Aims To compare the effectiveness of transient prehypertensive treatment with losartan versus amlodipine in spontaneously hypertensive rats (SHR) on long-term blood pressure and cardiac protection Main methods SHR were prehypertensively (weeks 4-10 of age) treated with losartan (SHR-Los: $20 \mathrm{mg} / \mathrm{kg} /$ day), amlodipine (SHR-Aml: $10 \mathrm{mg} / \mathrm{kg} /$ day) or saline ( $\mathrm{n}=24$ each group). Rats were followed up until week 46. Systolic blood pressure (SBP) was measured by tail-cuff method. Cardiac parameters including Left ventricular (LV) mass index (LVMI), collagen volume fraction (CVF) and LV function were assessed by histomorphometry and echocardiography. Plasma and myocardium angiotensin II (Ang II) and aldosterone (Aldo) were measured by radioimmunoassay. Cardiac angiotensin II type 1 and type 2 receptor (AT1R and AT2R) protein were determined by immunoblotting and brain natriuretic peptide (BNP) mRNA was semi-quantified by reverse transcription-PCR (RT-PCR).

Key findings The SBP in SHR-Los was reduced until age 46 weeks, but returned to untreated SHR levels in SHR-Aml from 30 weeks onwards. Compared to untreated SHR, the LVMI and CVF in SHRLos were markedly decreased until week 46, and the LV ejection fraction (LVEF) (SHR-Los vs SHR: $83.1 \pm 2.3 \%$ vs $79.5 \pm 1.9 \%$, $\mathrm{p}<0.05)$ and cardiac BNP mRNA expression were improved, whereas comparable LVMI and elevated CVF were found in SHRAml, and the LVEF fell significantly below that of untreated SHR at week 46 (SHR-Aml vs SHR: $74.4 \pm 4.3 \%$ vs $79.5 \pm 1.9 \%, p<0.05$ ), with cardiac BNP mRNA expression increasing slightly. Compared to untreated SHR, the plasma and myocardium Ang II and Aldo levels in SHR-Los at week 46 were remarkably decreased (plasma Ang II: $302 \pm 32$ vs $458 \pm 32 \mathrm{pg} / \mathrm{ml}$; plasma Aldo: $172 \pm 20$ vs $252 \pm 41 \mathrm{pg} / \mathrm{ml}$; cardiac Ang II: $126 \pm 11$ vs $199 \pm 14 \mathrm{pg} / 100 \mathrm{mg}$; cardiac Aldo: $497 \pm 43$ vs $766 \pm 46 \mathrm{pg} / 100 \mathrm{mg}$, all $\mathrm{p}<0.05$ ), and the cardiac AT1R protein was down-regulated and AT2R protein was up-regulated, no significant difference of these indices was found between SHR-Aml and untreated SHR. Significance Prehypertensive treatment with losartan was more effective than amlodipine on delaying long-term blood pressure rise and meliorating cardiac structure and function, which might be related to permanent attenuation of circulating and local renin-angiotensin (R-A) systems.

\section{e0159 OSTEOPONTIN IS INVOLVED IN UROTENSIN II INDUCED MIGRATION OF ADVENTITIAL FIBROBLASTS FROM RAT AORTA}

doi:10.1136/hrt.2010.208967.159

${ }^{1}$ Zhang Yonggang, ${ }^{1}$ Kuang Zejian, ${ }^{1}$ Mao Yanyan, ${ }^{2}$ Wei Ruihong, ${ }^{1}$ Bao Shilin, ${ }^{1}$ Wu Libiao, ${ }^{1} \mathrm{Hu}$ Yanchao. ${ }^{1}$ First Affiliated Hospital, Shantou University Medical College, Shantou, China; ${ }^{2}$ Second Affiliated Hospital, Shantou University Medical College, Shantou, China

Background Recent studies suggest osteopontin (OPN) plays a critical role in the progression of vascular remodelling, and that Urotensin II (UII) is a potent vasoconstrictor and stimulator of cellular migration. The goal of this study was to test the hypothesis that OPN is involved in UII-induced migration of rat aortic adventitial fibroblasts (AFs), and examine the effect and mechanisms of UII on OPN expression. 